Saudi Journal of Medical and Pharmaceutical Sciences

Abbreviated Key Title: Saudi J Med Pharm Sci ISSN 2413-4929 (Print) |ISSN 2413-4910 (Online) Scholars Middle East Publishers, Dubai, United Arab Emirates Journal homepage: https://saudijournals.com/sjmps

Case Report

\title{
Epithelial-Myoepithelial Carcinoma of the Parotid Gland
}

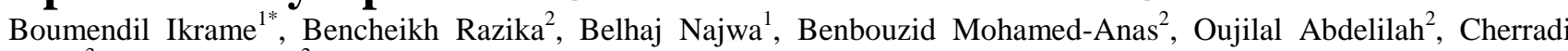
Nadia $^{3}$, Essakalli Leila ${ }^{2}$

${ }^{1}$ Resident Physician in Otorhinolaryngology, Department of Otorhinolaryngology, Head and Neck Surgery, Ibn Sina University Hospital, Faculty of Medicine, Mohammed V University, Rabat, Morocco

${ }^{2}$ Professor of otorhinolaryngology, Department of Otorhinolaryngology, Head and Neck Surgery, Ibn Sina University Hospital, Faculty of Medicine, Mohammed V University, Rabat, Morocco

${ }^{3}$ Professor of Pathological Anatomy, Department of Pathological Anatomy, Ibn Sina University Hospital, Faculty of Medicine, Mohammed V University, Rabat, Morocco

\author{
DOI: $10.36348 /$ sjmps.2020.v06i06.003 \\ | Received: 25.05.2020 | Accepted: 01.06.2020 | Published: 16.06.2020 \\ *Corresponding author: Boumendil Ikrame
}

\section{Abstract}

Epithelial-myoepithelial carcinoma (EMC) is an unusual malignant salivary gland tumor. Little is known about the clinical and biological behavior of EMC. There is no consensus for its treatment. It is considered as a low grade malignant tumor. Whatever, it presents a potential of local recurrence and possibility of metastases. We report a 76-yearold patient who presented an epithelial-myoepithelial carcinoma of parotid gland, treated successfully with wide local resection and adjuvant radiotherapy with no signs of recurrence for 3 years.

Keywords: Epithelial-myoepithelial carcinoma, parotid gland, surgery.

Copyright @ 2020: This is an open-access article distributed under the terms of the Creative Commons Attribution license which permits unrestricted use, distribution, and reproduction in any medium for non-commercial use (NonCommercial, or CC-BY-NC) provided the original author and source are credited.

\section{INTRODUCTION}

Epithelial-myoepithelial carcinoma (EMC) is a rare malignant tumor accounting about $1 \%$ for all salivary gland tumors [3]. It arises most commonly in the parotid gland, but it has also been described in the submandibular gland, minor salivary gland and palate [4]. EMC is composed by a dual cells population: central duct lining cells surrounded by clear cells of myoepithelial origin. In 1972, Donath and al introduce term of epithelial myoepithlial carcinoma [1]. The World Health organization (WHO) recognized EMC as a distinct entity in 1991 [2]. EMC is considered to be a low grade malignant tumor with high potential of recurrence and possibility of metastases. Treatment is mainly surgical [2].

We report a case about a 76-year-old patient treated in our department for an epithelial-moyepitheial carcinoma of parotid gland with no recurrence on 3 years' follow-up period.

\section{Case Report}

We present the case of a 76-year-old patient; followed for high blood pressure; admitted in our university hospital for a mass over the left parotid gland. This painless mass had been slowly enlarging for two years. At the physical examination, it appeared as a bulky mass of $6 \mathrm{~cm}$. It had a firm consistency, was fixed to the skin and was not painful on palpation. There was a facial paralysis stage $\mathrm{V}$ of HouseBrackmann classification of facial function. The oral cavity examination was normal. There were no palpable cervical lymph nodes on both sides.

A preoperative cervical computed tomography (CT) showed a left superficial parotid lobe tumor. It was well a limited, hypodense, heterogeneous, multilobed mass with irregular contours. It was containing micro-calcifications, and increasing heterogeneously after injection of contrast product. There were no cervical lymph nodes on CT either (Figure-1).

Our patient was classified $\mathrm{T}_{4} \mathrm{~N}_{0} \mathrm{M}_{0}$, then the treatment was as follow: a left exofacial parotidectomy was performed without lymph nodes dissection. The extemporaneous examination was inconclusive.

In the final pathology report, two components of the tumor cells including the luminal ductal inner epithelial cells and peripheral outer myoepithelial cells were revealed (Figure-4). The results of biphasic differentiation were confirmed by immunohistochemical staining: positive for S-100 and smooth muscle actin in the surrounding myoepithelial cells, and intense positive for epithelial markers, such 
Boumendil Ikrame et al; Saudi J Med Pharm Sci, June, 2020; 6(6): 438-442

as cytokeratin-7 (CK7) and CD99 in the luminal tumor cells. They are also negative for CD45, CD20, CD3, CD1O, bc16, MuM1. The Ki-67 (proliferation index) was less than $1 \%$ (Figure-5). Based on these results, the mass was diagnosed as an epithelial-myoepithelial carcinoma of the parotid gland. Postoperatively, the patient was treated with adjuvant radiotherapy

The follow-up was satisfying for 3 years without signs of recurrence.
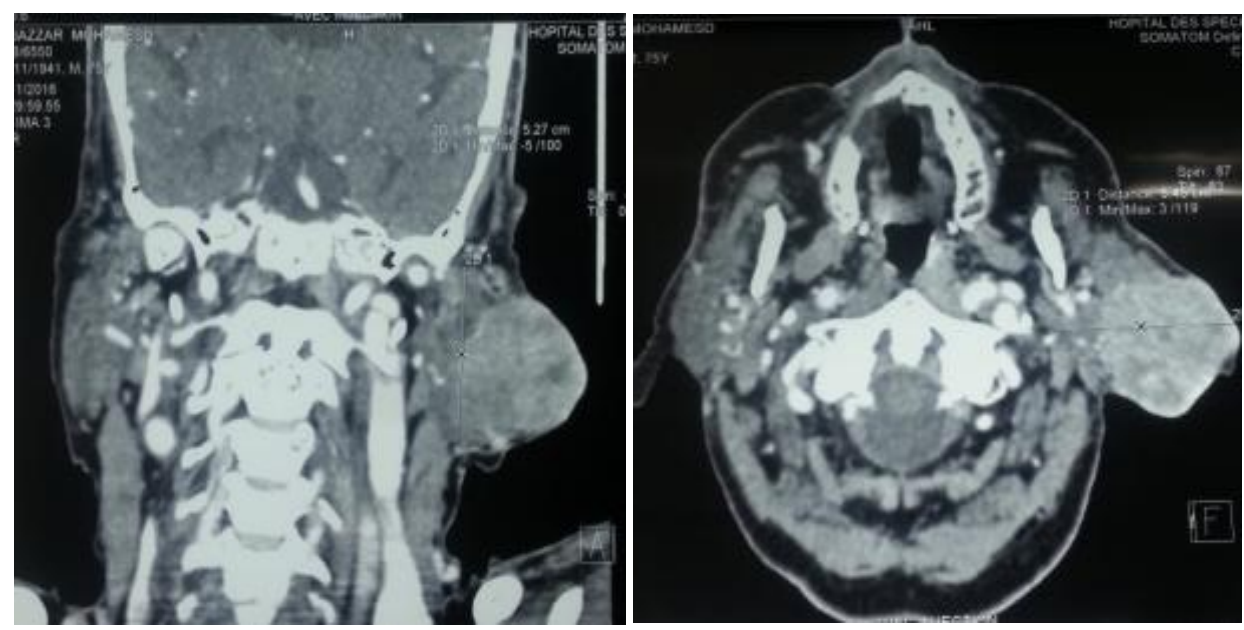

Fig-1: A computed tomography scan shows a non-homogeneously enhancing mass in the left parotid gland
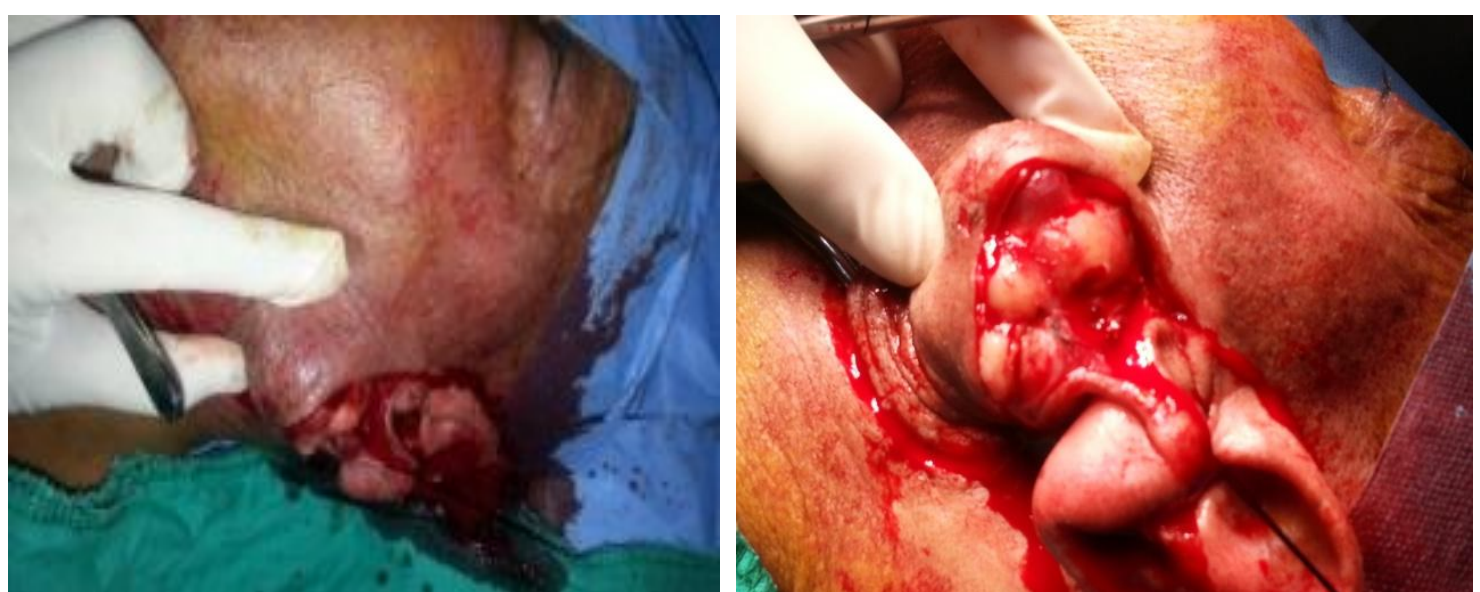

Fig-2: A per-operative Tumor mass $(6 \mathrm{~cm})$ in the left parotid with involvement of skin

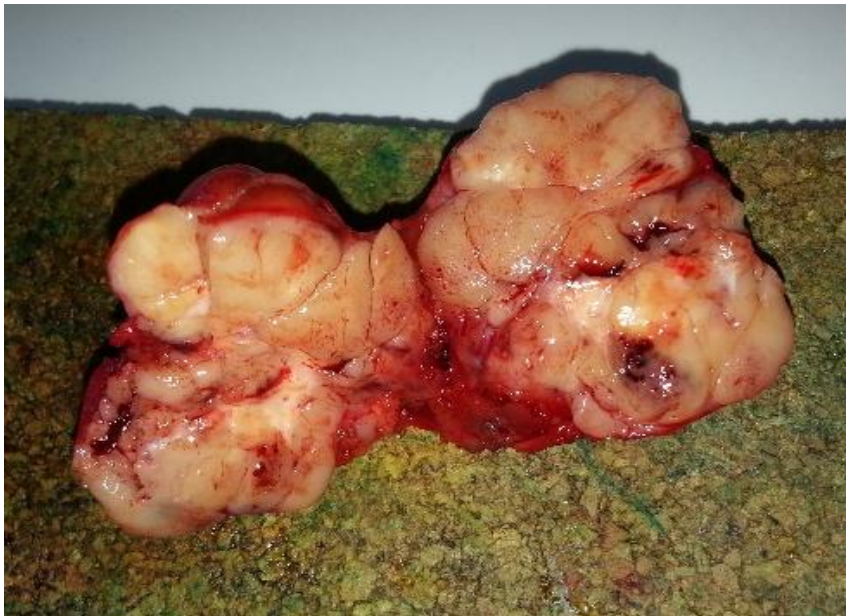

Fig-3: Macroscopic appearance of the resected tumor. The cut surface revealed a homogenous multilobated and gray-white appearance 

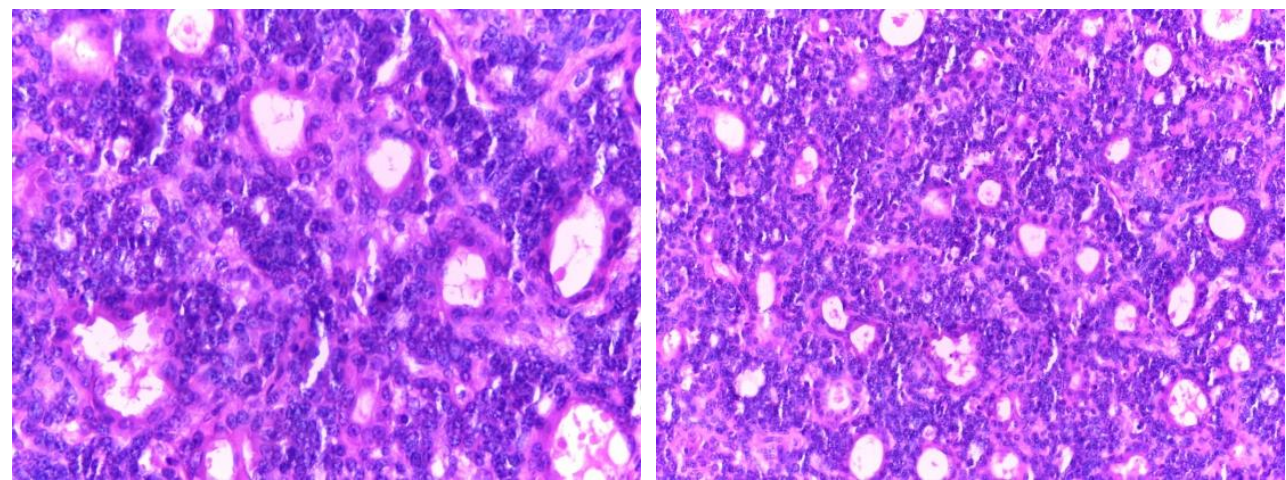

Fig-4: High-power microscopic observation of the hematoxylin-eosin-stained specimen shows a glandular structure consisting of inner layer (eosinophilic cuboidal cells) and outer layer (clear cells)

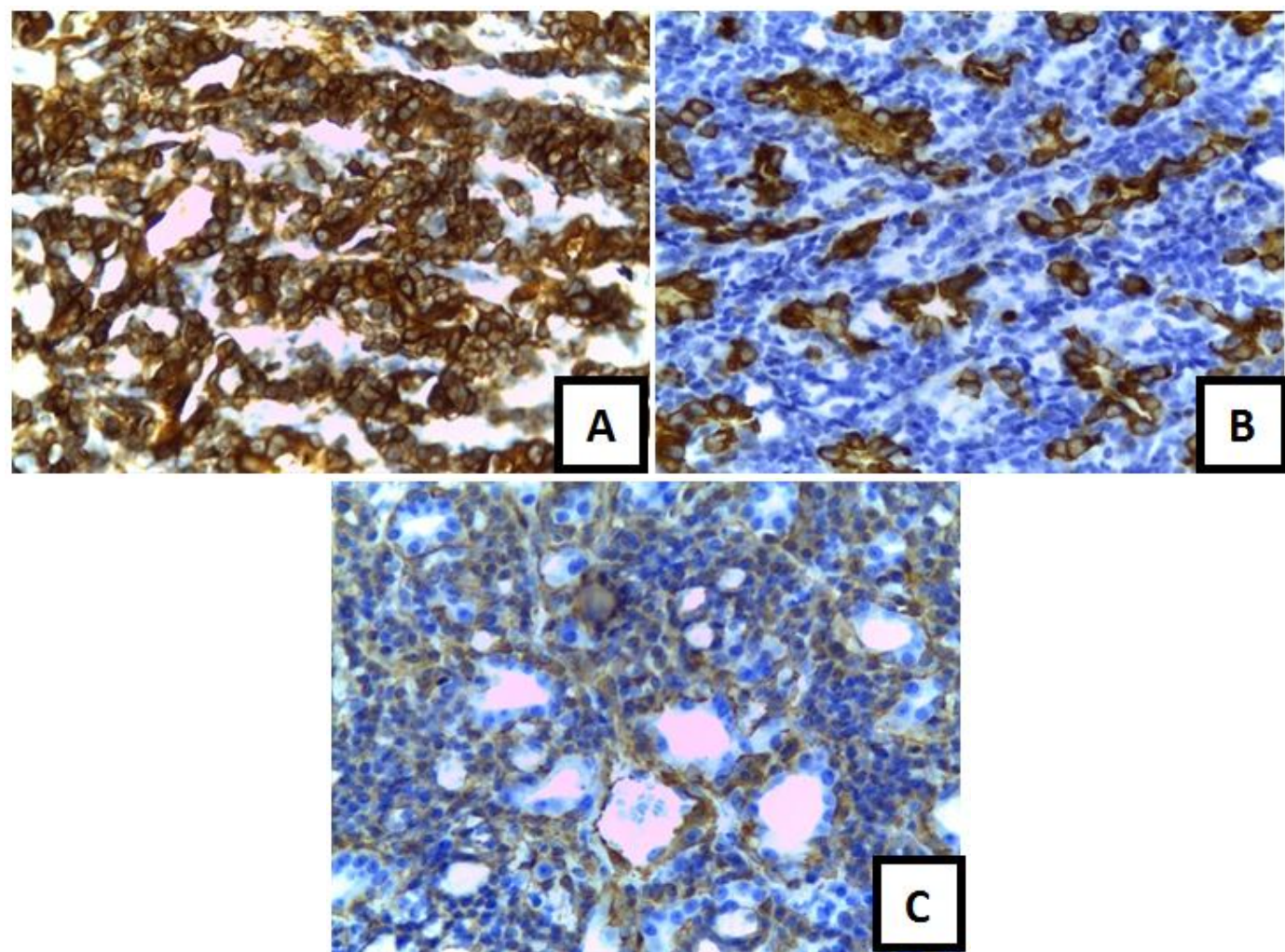

Fig-5: Immunohistochemical staining of sections: The inner-layer cells are positive for cytokeratin AE1/AE3 (A) and CK7 (B) as epithelial markers. The outer-layer cells are positive for actin (C) as a myoepithelial marker

\section{DISCUSSION}

Epithelial myoepithelial carcinoma (EMC) is a rare low grade malignant tumor, firstly described in 1972 by Donath et al., [1]. It was recognized as a distinct entity by the World Health Organization in $1991[2,3]$.

EMC accounts about $1 \%$ of all salivary gland tumors. It arises most commonly in the parotid gland $(60$ to $83 \%)[3,4]$ followed by the submandibular gland $(9.8$ to $13.01 \%)[5,4]$ and occasionally occurs in minor salivary gland and palate [5].

The peak of incidence appears in the sixth and seventh decades, with a female predominance (57.2 à $62.2 \%)[3,4]$.
Clinical presentation of EMC is similar to other parotid gland tumors. It may be presented by an asymptomatic painless slow growing mass suddenly increasing in size. Pain and facial paralysis can be associated. Radiological appearance (computed tomography (CT) and magnetic resonance imaging $(\mathrm{MRI}))$ of this tumor is non-specific [6,7].

According to literature, the majority of the patients had $\mathrm{T} 1$ (21.8 to $38.35 \%)$ or $\mathrm{T} 2$ (21.6 to $35.33 \%$ ) tumors. The nodal status of 3.76 to $4.2 \%$ of patients was $\mathrm{N}+$. The rate of distant metastases at diagnosis is about $2.6 \%[4,5]$. 
Table: Patient tumor (T), nodal (N), and metastasis (M) characteristics:

\begin{tabular}{|l|l|l|}
\hline & Vazquez 2015 [4] & Gore 2018 [5] \\
\hline T classification & & \\
T1 & $38.35 \%$ & $21.8 \%$ \\
T2 & $32.33 \%$ & $21.6 \%$ \\
T3 & $16.54 \%$ & $9.8 \%$ \\
T4 & $5.26 \%$ & $0.4 \%$ \\
Tx & $7.52 \%$ & $6.0 \%$ \\
\hline N classification & & \\
N0 & $90.23 \%$ & $57.5 \%$ \\
N1 & $3.76 \%$ & $3.8 \%$ \\
N2 & - & $0.4 \%$ \\
N3 & - & $0.2 \%$ \\
Nx & $6.02 \%$ & $4.7 \%$ \\
\hline M classification & & \\
M0 & $93.98 \%$ & $61.1 \%$ \\
M1 & - & $2.6 \%$ \\
Mx & $6.02 \%$ & $5.2 \%$ \\
\hline
\end{tabular}

Histopathologically, EMC is characterized by a glandular structure consisting of a double layer of inner and outer layers: the inner layer consists of intercalated duct epithelium-like cells; whereas the outer layer consists of clear neoplasic myoepithelial cells often predominate in number. [8]

The morphologic appearance of the neoplasm may mimic other clear cell salivary tumors as: clear cell carcinoma, acinar cell carcinoma, mucoepidermoid carcinoma, metastatic renal cell carcinoma, myoepithelial carcinoma, clear cell oncocytoma and pleomorphic adenoma. [8]

The diagnosis can be determined by immunohistochemical findings, because only innerlayer cells are positive for epithelial markers (EMA+, Keratin+) pancytokératine KL1 and only outer-layer cells are positive for myoepithelial markers (S100 protein + , Smooth muscle actin + , vimentin) [8].

Until now, there is no consensus about the treatment of EMC. Whatever, surgery appears to be the primary treatment of the EMC of parotid gland. Either exofacial or total parotidectomy is indicated to obtain a complete resection with negative margins even it is possible [3-5].

Based on nodal status, surgery was including neck dissections in $53.33 \%$ of the patients with N0 diseases, $80 \%$ with $\mathrm{N}+$ and $37.5 \%$ with $\mathrm{Nx}$ [4].

Adjuvant radiotherapy is also required. Its survival benefit still unclear, but it may be effective in preventing local recurrence [4]. The efficacy of adjuvant chemotherapy has not been established [9].

Indeed, the low-grade malignancy of this tumor, the recurrence rate was $36.3 \%$ (12/33 patient), with a median disease-free survival DFS of 11.34 years
[3]. Overall disease-specific survival was about $91.3 \%$, $90.2 \%$ and $80.7 \%$ at 60,120 and 180 months [4].

Different studies showed that overall survival was significantly affected by TNM stage (presence of nodal/distant metastases), surgical versus nonsurgical treatment, race and age at diagnosis [5].

\section{CONCLUSION}

Epithelial myoepithelial carcinoma is an unusual salivary gland tumor. The usual treatment is wide surgical resection with negative soft-tissue margins. The role of radiation and chemotherapy is still controversial. EMC is a low-grade malignant tumor with a potential of local recurrence and possibility of metastases. Therefore, more studies are needed to better understand the role of neck dissection and adjuvant therapy to affect survival and recurrence.

\section{REFERENCES}

1. Donath, K., Seifert, G., \& Schmitz, R. (1972). Zur Diagnose und Ultrastruktur des tubulären Speichelgangcarcinoms. Virchows Archiv A, 356(1), 16-31.

2. Angiero, F., Sozzi, D., Seramondi, R., \& Valente, M. G. (2009). Epithelial-myoepithelial carcinoma of the minor salivary glands: immunohistochemical and morphological features. Anticancer research, 29(11), 4703-4709.

3. Seethala, R. R., Barnes, E. L., \& Hunt, J. L. (2007). Epithelial-myoepithelial carcinoma: a review of the clinicopathologic spectrum and immunophenotypic characteristics in 61 tumors of the salivary glands and upper aerodigestive tract. The American journal of surgical pathology, 31(1), 44-57.

4. Vázquez, A., Patel, T. D., D’Aguillo, C. M., Abdou, R. Y., Farver, W., Baredes, S., ... \& Park, R. C. W. (2015). Epithelial-myoepithelial 
carcinoma of the salivary glands: an analysis of 246 cases. Otolaryngology--Head and Neck Surgery, 153(4), 569-574.

5. Gore, M. R. (2018). Epithelial-myoepithelial carcinoma: a population-based survival analysis. BMC Ear, Nose and Throat Disorders, 18(1), 15.

6. Tauziede-Espariat, A., Raffoul, J., Sun, S. R., Monnin, C., Lassabe, C., \& Costes, V. (2015, December). A bilateral epithelial myoepithelial carcinoma of the parotid gland. In Annales de pathologie, 35(6), 489-495.

7. Yu, G. Y., Ma, D. Q., Zhang, Y., Peng, X., Cai, Z. G., Gao, Y., \& Chen, Y. (2004). Multiple primary tumours of the parotid gland. International journal of oral and maxillofacial surgery, 33(6), 531-534.

8. Colella, G., Giudice, A., Adolfo, A., De Simone, S., Rossiello, L., \& Rossiello, R. (2004). Epithelial-myoepithelial carcinoma of the parotid gland. Oral Oncology Extra, 40(2), 29-32.

9. Yamazaki, H., Ota, Y., Aoki, T., \& Kaneko, A. (2013). Lung metastases of epithelialmyoepithelial carcinoma of the parotid gland successfully treated with chemotherapy: a case report. Journal of Oral and Maxillofacial Surgery, 71(1), 220-226. 